\title{
Location estimation using RSS measurements with unknown path loss exponents
}

\author{
Musa Bora Zeytinci, Veli Sari, Frederic Kerem Harmanci^, Emin Anarim and Mehmet Akar
}

\begin{abstract}
The location of a mobile station (MS) in a cellular network can be estimated using received signal strength (RSS) measurements that are available from control channels of nearby base stations. Most of the recent RSS-based location estimation methods that are available in the literature rely on the rather unrealistic assumption that signal propagation characteristics are known and independent of time variations and the environment. In this paper, we propose an RSS-based location estimation technique, so-called multiple path loss exponent algorithm (RSS-MPLE), which jointly estimates the propagation parameters and the MS position. The RSS-MPLE method incorporates antenna radiation pattern information into the signal model and determines the maximum likelihood estimate of unknown parameters by employing the Levenberg-Marquardt method. The accuracy of the proposed method is further examined by deriving the Cramer-Rao bound. The performance of the RSS-MPLE algorithm is evaluated for various scenarios via simulation results which confirm that the proposed scheme provides a practical position estimator that is not only accurate but also robust against the variations in the signal propagation characteristics.
\end{abstract}

Keywords: Mobile positioning; Location estimation; Cramer-Rao bound; Received signal strength

\section{Introduction}

Recently, location estimation has been among the most attractive research topics in the area of cellular communications. With accurate position estimation, a variety of applications and services such as emergency services, monitoring and tracking fraud protection, asset tracking, fleet management, mobile yellow pages, and even cellular system design and management can become feasible for cellular networks [1]. These potential applications of wireless positioning have also been recognized by the IEEE, which set up a standardization group 802.15.4a for designing a new physical layer for low-data rate communications combined with positioning capabilities [2]. Furthermore, the Federal Communications Commission (FCC) in the USA has required wireless providers to locate mobile users within tens of meters for emergency 911 calls [3].

The position of a mobile station (MS) can be determined using multiple radio signals transmitted or received by the MS. Some location estimation methods like assisted

\footnotetext{
*Correspondence: mehmet.akar@boun.edu.tr

'Deceased

Department of Electrical and Electronics Engineering, Bogazici University, Bebek, Istanbul, Turkey
}

global positioning system (A-GPS) are based on signals transmitted from satellites, while others rely on measurements of signals between MS and base stations (BS), the so-called network-based methods. Currently, the best positioning accuracy in cellular systems is provided by AGPS at the expense of a significant increase in network and handset complexity [4]. For instance, modifications on the handset such as an embedded GPS receiver and deployment of location management units (LMU) into the network are needed in order to operate A-GPS systems [5]. Compared to A-GPS, network-based methods are relatively less complex. Moreover, they can be used in many situations where the A-GPS method cannot be applied, i.e., indoor positioning, but generally with a degradation in accuracy. So far, a wide variety of network-based positioning techniques have been proposed which use measurements obtained within the cellular networks, such as received signal strength (RSS), time of arrival (TOA), time difference of arrival (TDOA), and angle of arrival (AOA) methods [6-15].

Positioning technology is often based on trilateration in time-based methods like TOA and TDOA, in which the MS position is obtained as the intersection point of

\section{Springer}

(c) 2013 Zeytinci et al. licensee Springer. This is an Open Access article distributed under the terms of the Creative Commons Attribution License (http://creativecommons.org/licenses/by/2.0), which permits unrestricted use, distribution, and reproduction in any medium, provided the original work is properly cited. 
three circles constituted by distance estimates [16]. Assurance of proper operation of time-based methods requires the deployment of LMUs [17]. These network elements perform timing measurements of all local transmitters, based on which the actual relative time difference for each BS can be estimated. In a similar manner, AOAbased positioning methods require the implementation of adaptive array antennas, by which the direction of signal arrival can be estimated. At the same time, the handset typically requires software modifications to enable positioning functionality with such a method. Consequently, these methods are not widespread in commercial systems due to their high deployment costs. Therefore, improving the accuracy of positioning systems based on the existing cellular network infrastructure is desired, which is the main motivation of this study.

The system model that is used for location estimation in this paper is based on RSS measurements. One fundamental MS function is to find the BSs with the strongest signal strength for cell selection purposes. Thus, RSSbased methods can be implemented without any hardware enhancement in either the MS or the BS. Distance information between MS and BS can be extracted from RSS measurements using accurate information about the propagation characteristics of the measurement channel. However, in most of the RSS-based location estimation techniques in the literature, it is assumed that the propagation parameters of the measurement channel are accurately known a priori, either through a training period or by assuming a perfect free-space channel condition [18-24]. Such an approach results in degraded positioning accuracy in many practical application scenarios, including mobile tracking techniques in $[25,26]$. More specifically, both $[25,26]$ study Monte Carlo-based mobility tracking algorithms under the assumption that the mobile moves in a $2 \mathrm{D}$ environment with input as acceleration. In [25], RSS data are used to predict mobile position and velocity using particle filtering techniques, but the method relies on known propagation parameters. In [26], the so-called interacting multiple model method has been extended to predict the state of the mobile for indoor applications by considering multiple fixed environment parameters at the expense of an increase in the number of random processes, whereas the technique herein relies on continuous parameter adaptation.

In this paper, a method that aims to resolve major shortcomings of the existing RSS-based positioning techniques is proposed. In particular, an RSS-based location estimation technique that jointly estimates the propagation parameters and the MS position is explained in Section 2. The Cramer-Rao bound (CRB) derivation and accuracy evaluation are given in Section 3 as a benchmark for performance comparison. In Section 4, simulation results under various scenarios are presented to evaluate the performance of the proposed algorithm. Finally, some concluding remarks are given in Section 5.

\section{RSS-based location estimation model}

Using RSS together with a path loss (PL) and shadow fading model, a distance estimate between the BS and the MS can be obtained. The propagation model used throughout this paper is a modified version of the log normal model that is widely used in the literature [27-30]. The PL exponent (PLE) is the key parameter in the log normal model. An accurate value of the PLE is required in order to obtain an accurate estimate of the MS-BS distance from the corresponding RSS measurement.

In most of the existing studies of RSS-based location estimation techniques in the literature, the channel model is assumed to be known a priori, that is, the path loss characteristics of the coverage area are considered known, either by assuming that the environment is a perfect free space or by extensive measurement and modeling prior to the deployment of location estimation systems. However, the PLE parameter is environment dependent $[31,32]$. Even in the same environment, propagation characteristics may change considerably over a long period of time, e.g., due to seasonal and/or weather changes [33]. In [32], it is experimentally demonstrated in an omnidirectional antenna system that the PLE is strongly dependent on the base antenna height and the terrain category. Extension of the experimental study in [32] to directional antenna systems can be found in [34] where the authors show that antenna beamwidth has an additional gain reduction influence on the PLE. In other experimental studies that consider path loss modeling for directional antenna systems $[35,36]$, the authors compute different PLEs for different areas of the cell that consist of different terrains (but not different PLEs for different directional antennas of the same base station). There are also related studies that utilize single PLE for directional antenna systems [37-39].

The proposed algorithm in this paper estimates the MS location using the available RSS measurements without any need for a training period or any need for the knowledge of the PLE value in the path loss model. The PLE values are determined and calibrated in real time for every mobile using the RSS measurements. By incorporating the antenna radiation patterns of the sectors in a BS into the signal model, an additional improvement in position estimates is provided. In determining the position of a given mobile user, it is assumed that the PLE is the same for all sectors of a given base station, whereas the PLE might be different for another. This is a quite realistic assumption in light of the discussion of the related literature in the previous paragraph, since the mobile user is in the same location with respect to the base station and directional antennas are at the same height. 


\subsection{Antenna model}

Point to multipoint and cellular communication systems commonly use fan-beam antennas for sector coverage. An approximate formula describing the normalized azimuth radiation pattern of the fan-beam antenna using three parameters is given by:

$$
A(\phi)=\exp \left[-a_{3 \mathrm{~dB}}\left(\frac{|\phi|}{\phi_{\mathrm{HBW}}}\right)^{\tau}\right] \text {, }
$$

where $a_{3 \mathrm{~dB}}$ is a unitless parameter that determines the pattern value at half power bandwidth $(\mathrm{HBW})|\phi|=\phi_{\mathrm{HBW}}$ (in radians) [40], i.e.,

$$
a_{3 \mathrm{~dB}}=-\ln \left(\frac{1}{\sqrt{2}}\right)=0.3465
$$

and $\tau$ is used to match the pattern to a second field value, $v$, at $|\phi|=\left|\phi_{v}\right|$ where $0<v<1$, i.e.,

$$
\tau=\frac{\ln \left(\frac{-\ln (v)}{a_{3 \mathrm{~dB}}}\right)}{\ln \left(\frac{\left|\phi_{v}\right|}{\phi_{3 \mathrm{~dB}}}\right)}
$$

In this paper, $\tau$ is evaluated at $\left|\phi_{v}\right|=\pi / 2$, and the value of $v$ is taken to be equal to 0.006 that is the inverse of the front-to-side ratio of the normalized pattern and $\phi_{\mathrm{HBW}}=\pi / 3$ is the recommended value according to ETSI EN 301 215-2 Class CS 2 requirement in a local multipoint distribution service point-to-multipoint application [40] (see Figure 1 for the radiation pattern used in this paper).

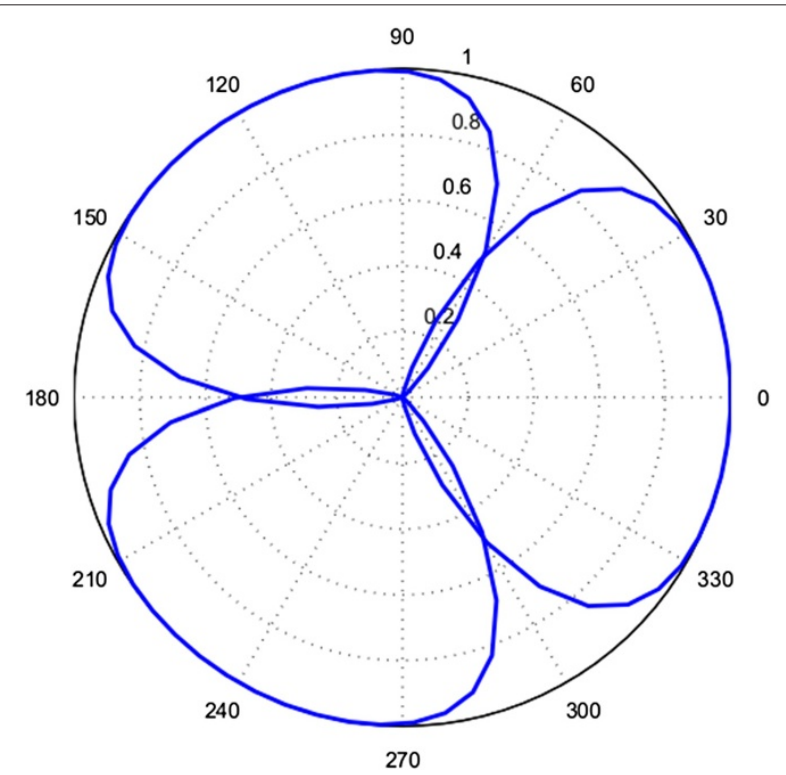

Figure 1 Normalized radiation pattern for 3-sector BS configuration.

\subsection{Path loss model}

In cellular networks, an MS measures received signal code power on a common pilot channel $(\mathrm{CPICH})$ in Universal Mobile Telecommunications System (UMTS) and RSS on a broadcast control channel (BCCH) in GSM systems to determine the received signal level. Signal strength measurements are indexed with base station and sectoral identifiers $k$ and $s$, respectively, in (4) [18] considering that transmitting cells use different channels (CPICHs or $\mathrm{BCCHs):}$

$$
R_{k, s}\left(x, y, \alpha_{k}\right)=\frac{P G A_{k, s}^{2}(x, y)}{\beta\left(\frac{d_{k, s}(x, y)}{d_{0}}\right)^{\alpha_{k}}} 10^{-v_{k, s} / 10} .
$$

In (4), $R_{k, s}$ is the received signal strength from sector $s$ of base station $k$ at point $(x, y)$ (in watts); $P$ is the total transmitted power on each sector $s$ (in watts), and $A_{k, s}(x, y)$ and $G$ are the normalized radiation pattern and the antenna gain in azimuth direction, respectively, that belongs to the corresponding cell of sector $s$; $d_{0}$ (in meters) is the free-space reference distance; $d(x, y)$ (in meters) is the distance between MS and BS at point $(x, y) ; v_{k, s}$ accounts for shadow fading; $\alpha_{k}$ is the PLE that is specific for $k$ th base station; and $A_{k, s}(\phi)$ is represented as $A_{k, s}(x, y)$ since it is possible to determine $\phi$ with the knowledge of MS and BS coordinates. Herein, it is assumed that $P, G$, and $\beta$ are assumed to be known $a$ priori and $\beta$ is calculated by [32]:

$$
\beta=\left(\frac{4 \pi d_{0}}{\lambda}\right)^{2}
$$

where $\lambda$ is the wavelength in meters and $d_{0}$ is chosen to be equal to $1 \mathrm{~m}$ in a microcell environment [31]. Rewriting the RSS in decibel $(\mathrm{dB})$, we have:

$$
\begin{aligned}
\mu_{k, s(\mathrm{~dB})}(\theta)= & 10 \log _{10}\left(\frac{P G}{\beta}\right)+20 \log _{10} A_{k, s}(x, y) \\
& -10 \alpha_{k} \log _{10} \frac{d_{k, s}(x, y)}{d_{0}} \\
& R_{k, s(\mathrm{~dB})}(\theta)=\mu_{k, s(\mathrm{~dB})}(\theta)-v_{k, s}
\end{aligned}
$$

where $\theta=\left[\begin{array}{lllll}x & y & \alpha_{1} & \ldots & \alpha_{k}\end{array}\right]^{T}$.

In this paper, it is assumed that the channels used by distant BSs may have different propagation characteristics. More specifically, the channels used by the cells that are served by the same BS are assumed to have the same PLE, and this PLE is not necessarily the same for other channels. Hence, the value of $R_{k, s}$ and $\mu_{k, s}$ depends on $\alpha_{k}$ in addition to the mobile position, $(x, y)$. 
2.3 Problem formulation and the positioning algorithm Based on the path loss model described above, the distribution of $R_{k, s(\mathrm{~dB})}(\theta)$ is Gaussian, i.e., we have:

$f_{R_{k, s(\mathrm{~dB})}}\left(R_{k, s(\mathrm{~dB})} ; \theta\right)=\frac{1}{\sigma_{v} \sqrt{2 \pi}} \exp \left[-\frac{\left(R_{k, s(\mathrm{~dB})}-\mu_{k, s(\mathrm{~dB})}(\theta)\right)^{2}}{2 \sigma_{v}{ }^{2}}\right]$,

where $f_{R_{k, s(\mathrm{~dB})}}\left(R_{k, s(\mathrm{~dB})} ; \theta\right)$ denotes the pdf for the deterministic unknown parameter $\theta$ [41]. Assuming $R_{k, s(\mathrm{~dB})}$ is independent and identically distributed (i.i.d.), the likelihood function, $L(\theta)$ can be written as:

$$
L(\theta)=\prod_{k=1}^{n} \prod_{s=1}^{m} f_{R_{k, s(\mathrm{~dB})}}\left(R_{k, s(\mathrm{~dB})} ; \theta\right)
$$

where $n$ is the number of BSs that have RSS measurements for the MS and $m$ is the number of sectors for each BS. Taking into account that $R_{k, s(\mathrm{~dB})}$ is an i.i.d. Gaussian random variable, $L(\theta)$ becomes:

$$
L(\theta)=\frac{1}{\sigma_{v} \sqrt{2 \pi}} \exp \left[-\frac{\sum_{k=1}^{n} \sum_{s=1}^{m}\left(R_{k, s(\mathrm{~dB})}-\mu_{k, s(\mathrm{~dB})}(\theta)\right)^{2}}{2 \sigma_{v}^{2}}\right] .
$$

In order to obtain the maximum likelihood (ML) estimate, $\theta_{\mathrm{ML}}$ that best approximates the available data, $L(\theta)$ should be maximized over $\theta$ (see [41], pp. 177). This in turn can be done by computing:

$$
\theta_{\mathrm{ML}}:=\arg \min _{\theta} \sum_{k=1}^{n} \sum_{s=1}^{m}\left(R_{k, s(\mathrm{~dB})}-\mu_{k, s(\mathrm{~dB})}(\theta)\right)^{2}
$$

where $\arg \min$ is the value of $\theta$ for which the given function is minimized over the given data set. Note that a distinctive advantage of ML estimate is that it can always be found for a given data set [41].

In this paper, we obtain this estimate using a recursive solution of a nonlinear least squares problem. To this end, define $\mathcal{J}(\theta)$ to be the cost function (which is to be minimized so that $L(\theta)$ in (9) is minimized):

$$
\mathcal{J}(\theta)=\sum_{k=1}^{n} \sum_{s=1}^{m}\left(R_{k, s(\mathrm{~dB})}-\mu_{k, s(\mathrm{~dB})}(\theta)\right)^{2}
$$

and

$$
\mathbf{f}(\theta)=\mathbf{R}-\mu(\theta),
$$

where $\mathbf{R}=\left[\begin{array}{llll}R_{1,1}, & R_{1,2}, \ldots R_{n, m-1}, & R_{n, m}\end{array}\right]^{T}$ and $\mu(\theta)=$ $\left[\begin{array}{llll}\mu_{1,1}, & \mu_{1,2}, \ldots, & \mu_{n, m-1}, & \mu_{n, m}\end{array}\right]^{T}, \mathcal{J}(\theta)$ can be represented in vector form as:

$$
\mathcal{J}(\theta)=(\mathbf{R}-\mu(\theta))^{T}(\mathbf{R}-\mu(\theta))=\mathbf{f}(\theta)^{T} \mathbf{f}(\theta)
$$

Thus, any $\theta^{*}$ that satisfies (14) is a solution of (10) if $\mathbf{f}^{\prime}\left(\theta^{*}\right)$ is nonsingular, i.e.

$$
0=\mathcal{J}^{\prime}\left(\theta^{*}\right)=2 \mathbf{f}^{\prime}\left(\theta^{*}\right)^{T} \mathbf{f}\left(\theta^{*}\right)
$$

In this paper, the Levenberg-Marquardt (LM) method which is a modified version of the Gauss-Newton method is employed to solve the nonlinear least squares problem $[42,43]$. The proposed algorithm which is summarized as Algorithm 1 on the next page employs a linear approximation of the nonlinear equations to find a least squares estimate of these equations iteratively. The vector $\boldsymbol{\mu}(\boldsymbol{\theta})$ that consists of nonlinear functions is linearized using the Taylor series expansion in which the second-order terms are omitted:

$$
\mu_{k, s(\mathrm{~dB})}(\theta) \approx \mu_{k, s(\mathrm{~dB})}\left(\theta^{(0)}\right)+J^{(0)}\left(\theta-\theta^{(0)}\right) .
$$

First-order Taylor series expansion is obtained for $\mu_{k, s(\mathrm{~dB})}(\theta)$ where $\theta^{(0)}$ is the initial estimate for $\theta_{\mathrm{ML}}$ and $J^{(0)}$ is the Jacobian matrix of $\mu_{k, s(\mathrm{~dB})}(\theta)$ at $\theta^{(0)}$. Consequently, the LM step size $\Delta$ at each iteration is obtained by solving:

$$
\left(\mathbf{J}^{T} \mathbf{J}+h \mathbf{I}\right) \Delta=\mathbf{J}^{T} \mathbf{f}
$$

where $h>0$, e.g., for $n=2$ BSs and $m=3$ sectors for each $\mathrm{BS}$, in case $\theta=\left[x, y, \alpha_{1}, \alpha_{2}\right]$ are unknown parameters, the Jacobian matrix J can be represented as:

$$
\mathbf{J}=\left[\begin{array}{cccc}
\frac{\partial\left(C_{11}(\theta)\right)}{\partial x} & \frac{\partial\left(C_{11}(\theta)\right)}{\partial y} & \frac{\partial\left(10 \alpha_{1} \log \left(d_{1}(\theta)\right)\right)}{\partial \alpha_{1}} & 0 \\
\frac{\partial\left(C_{12}(\theta)\right)}{\partial x} & \frac{\partial\left(C_{12}(\theta)\right)}{\partial y} & \frac{\partial\left(10 \alpha_{1} \log \left(d_{1}(\theta)\right)\right)}{\partial \alpha_{1}} & 0 \\
\frac{\partial\left(C_{13}(\theta)\right)}{\partial x} & \frac{\partial\left(C_{13}(\theta)\right)}{\partial y} & \frac{\partial\left(10 \alpha_{1} \log \left(d_{1}(\theta)\right)\right)}{\partial \alpha_{1}} & 0 \\
\frac{\partial\left(C_{21}(\theta)\right)}{\partial x} & \frac{\partial\left(C_{21}(\theta)\right)}{\partial y} & 0 & \frac{\partial\left(10 \alpha_{2} \log \left(d_{2}(\theta)\right)\right)}{\partial \alpha_{2}} \\
\frac{\partial\left(C_{22}(\theta)\right)}{\partial x} & \frac{\partial\left(C_{22}(\theta)\right)}{\partial y} & 0 & \frac{\partial\left(10 \alpha_{2} \log \left(d_{2}(\theta)\right)\right)}{\partial \alpha_{2}} \\
\frac{\partial\left(C_{23}(\theta)\right)}{\partial x} & \frac{\partial\left(C_{23}(\theta)\right)}{\partial y} & 0 & \frac{\partial\left(10 \alpha_{2} \log \left(d_{2}(\theta)\right)\right)}{\partial \alpha_{2}}
\end{array}\right],
$$

where $C(\theta)_{k s}=20 \log \left(A_{k s}(\theta)\right)-10 \alpha_{k} \log \left(d_{k}(\theta)\right)$ and $d_{k}$ represents the distance between $k$ th BS and the MS, that is, $d_{k}(\theta)=\sqrt{\left(x-x_{k}\right)^{2}+\left(y-y_{k}\right)^{2}}$. Herein, $k$ index represents the BS, and $s$ represents the sector that belongs to BS $k$. Since each channel used by different BSs may have a distinct PLE, $\alpha$ parameters are indexed with BS ID, $k$.

In Algorithm $1, k_{\max }$ is the maximum number of allowed iterations ( $k_{\max }=400$ is used in the simulations), and $\epsilon_{1}$ is used to detect how close the estimate is to the desired value (e.g., $\epsilon_{1}=10^{-15}$ ). Both parameters are chosen by the user. The damping parameter of the LM algorithm, $h$, is positive, which guarantees that $\Delta$ is a descent direction. Note that for large values of $h$, we have $\Delta=$ $J^{T}(\theta) f(\theta) / h$, which implies a short step in the descent direction, which in turn is good if the current iterate is far from the solution. On the other hand, if $h$ is small, then $\Delta$ is approximately equal to what we have from the Gauss-Newton iteration. Since the damping parameter 
Algorithm 1 LM-based algorithm for joint estimation of propagation parameters and mobile position

1: (Initialization)

(i) Let $d_{1}$ be the initial radial distance estimate obtained using Cell ID + Timing advance (Cell ID + Round trip time in UMTS case). (Both of these measurements are available from the serving cell.)

(ii) Let the number of RSS measurements from different sectors of the same BS be $n_{s}$. If $n_{s}=1$, then let $\phi$ be the azimuth angle of the serving sector else if $n_{s}>1$, utilize the LM method (as in step 3 below) with $\theta=\left[\phi, \alpha_{1}\right]$ and $J(\theta)$ as follows:

$$
\mathbf{J}(\theta)=\left[\begin{array}{ll}
\frac{\partial\left(20 \log \left(A_{11}(\phi)\right)\right)}{\partial \phi} & \frac{-10 \alpha_{1} \log \left(d_{1}\right)}{\partial \alpha_{1}} \\
\frac{\partial\left(20 \log \left(A_{12}(\phi)\right)\right)}{\partial \phi} & \frac{-10 \alpha_{1} \log \left(d_{1}\right)}{\partial \alpha_{1}} \\
\frac{\partial\left(20 \log \left(A_{13}(\phi)\right)\right)}{\partial \phi} & \frac{-10 \alpha_{1} \log \left(d_{1}\right)}{\partial \alpha_{1}}
\end{array}\right],
$$

where $\phi$ is the angle between serving BS and MS in polar coordinates and $A_{1 s}(\phi)$ is the radiation pattern of the $s$ th sector of the serving BS.

$\phi$ together with $d_{1}$ provides an initial estimate of the MS position in polar coordinates.

2: If the number of RSS measurements is greater than or equal to $n+2$ where at least one measurement is available from each of the BSs, then let $\theta=\left[x, y, \alpha_{1}, \alpha_{2}, \ldots, \alpha_{n}\right]$ (this is what we call the RSS multipath loss exponent (RSS-MPLE) algorithm in this paper); otherwise, let $\theta=\left[x, y, \alpha_{1}\right]$ (in this case, we can compute a single-path loss exponent, and we refer to this algorithm as the RSS single-path loss exponent (RSS-SPLE) algorithm).

3: LM method is employed to solve the stated nonlinear least squares problem iteratively.

(i) Set $k=0, \theta=\theta(k), h=\max \operatorname{diag} \mathbf{J}^{T}(\theta) \mathbf{J}(\theta)$.

(ii) While $\left(k \leq k_{\max }\right)$ and $\left(\left\|\mathbf{J}^{T}(\theta) \mathbf{f}(\theta)\right\|>\epsilon_{1}\right)$

Set $k=k+1$ and solve $\Delta$ from $\left(\mathbf{J}^{T}(\theta) \mathbf{J}(\theta)+h \mathbf{I}\right) \Delta=\mathbf{J}^{T}(\theta) \mathbf{f}(\theta)$

Compute $\theta_{\text {new }}=\theta-\Delta$

Compute the step size $\rho=\left(F(\theta)-F\left(\theta_{\text {new }}\right)\right) /\left(0.5 \Delta^{T}(h \Delta-f(\theta))\right)$

If $\rho>0$ (i.e., step acceptable), then set $\theta=\theta(k)=\theta_{\text {new }}$, and

$h=h \max \{1 / 100, h / 10\}$

else set $h=2 h$

end

influences both the direction and the size of the step, its update is controlled by the gain ratio $\rho$ in the algorithm. A large positive value of $\rho$ indicates a good approximation which allows us to decrease $h$ so that the LM step is closer to the Gauss-Newton step, whereas a small or negative $\rho$ is a poor approximation which requires an increase of damping by twofold in order to get closer to the steepest direction and hence increase chances of faster convergence. By this choice of parameters similar to [44], we have observed linear to superlinear convergence in our problem, although it is harder to make specific statements on the convergence rate for the problem in hand. However, it is well known that the Levenberg-Marquardt method has a quadratic rate of convergence when Jacobian is a nonsingular square matrix and if the parameter is chosen suitably at each step. The condition of the nonsingularity of Jacobian is too strong, and it is not valid in our problem either. Although the authors show in $[42,43]$ that the method has quadratic convergence under appropriate assumptions and the choice of the damping parameter, the results are valid only locally. In the next section, we derive the CRB bounds for the proposed method.

\section{The Cramer-Rao lower bound}

The CRB for RSS estimation depends on the strength of signal, Gaussian random variable and path loss exponent. In radio propagation channel studies, the random variable $v$ in the path loss model (4) is considered a zeromean Gaussian random variable, i.e., $N\left(0, \sigma_{v}^{2}\right)$, while its standard deviation $\sigma_{v}^{2}$ depends on the characteristics of a specific environment [45]. In the computation of the Cramer-Rao bound, we let $p_{k, s}=10 \log _{10} P G / \beta-R_{k, s}$, which is the observed path loss in decibel from $1 \mathrm{~m}$ to $d$ meters. Thus, the system of nonlinear equations for location estimation can be rewritten as [46]:

$$
p_{k, s}=g_{k, s}(\theta)+v, \quad 1 \leq k \leq n, \text { and } 1 \leq s \leq 3,
$$

where $g_{k, s}(\theta)=10 \alpha_{k} \log _{10} d_{k}-20 \log _{10} A_{k, s}(\phi)$ and the unknown vector parameter $\theta=\left[\begin{array}{lll}x & y & \alpha_{1} \ldots \alpha_{k}\end{array}\right]^{T}, k$ is the 
index identifying the base stations, while $s$ is the index representing the antenna sector. For each base station $k$, there are three antenna sectors $s=1,2,3$ defined. In this setting, the path loss observation $p_{k}$ defined in (19) has a probability density function:

$$
f\left(p_{k, s} ; \theta\right)=\frac{1}{\sqrt{2 \pi} \sigma_{v}} \exp \left(-\frac{\left(p_{k, s}-g_{k, s}(\theta)\right)^{2}}{2 \sigma_{v}^{2}}\right),
$$

which is parameterized by the unknown vector parameter $\theta$. If we assume that $p_{k, s}, 1 \leq k \leq n, 1 \leq s \leq$ 3 are statistically independent observations (which is a reasonable assumption as the transmitted powers are independent), the joint distribution of observation vector $\mathbf{p}=\left[p_{1,1}, p_{1,2}, p_{1,3}, \ldots, p_{n, 1}, p_{n, 2}, p_{n, 3}\right]$ is obtained as:

$$
f(\mathbf{p} ; \theta)=\prod_{k=1}^{n} \prod_{s=1}^{3} f\left(p_{k, s} ; \theta\right) \text {. }
$$

The CRB on the covariance matrix of any unbiased estimator $\hat{\theta}$ is defined as:

$$
\operatorname{cov}(\hat{\theta})-\mathbf{F}^{-1} \geq 0
$$

where $\mathbf{F}=-E\left[\Delta_{\theta}\left(\Delta_{\theta} \ln f(\mathbf{p} ; \theta)\right)^{T}\right]$ is the Fisher information matrix (FIM) [41].

Given the joint distribution of the observation vector $\mathbf{p}$ in (21), the equivalent log-likelihood function can be defined as:

$$
l(\theta)=-\frac{1}{2 \sigma_{v}^{2}} \sum_{k=1}^{n} \sum_{s=1}^{3}\left(p_{k, s}-g_{k, s}(\theta)\right)^{2},
$$

from which the Fisher information matrix can be derived as:

$$
\begin{aligned}
& F_{i j}=[\mathbf{F}]_{i j}=-E\left[\frac{\partial^{2} l(\theta)}{\partial \theta_{i} \partial \theta_{j}}\right] \\
& = \begin{cases}\frac{1}{\sigma_{v}^{2}} \sum_{k=1}^{n} \sum_{s=1}^{3}\left(\frac{\partial g_{k, s}(\theta)}{\partial \theta_{i}}\right)^{2} & \text { if } i=j ; \\
\frac{1}{\sigma_{v}^{2}} \sum_{k=1}^{n} \sum_{s=1}^{3} \frac{\partial g_{k, s}(\theta)}{\partial \theta_{i}} \frac{\partial g_{k, s}(\theta)}{\partial \theta_{j}} & \text { if } i \neq j .\end{cases}
\end{aligned}
$$

Recall that $g_{k, s}(\theta)$ is defined by $g_{k, s}(\theta)=10 \alpha_{k} \log _{10} d_{k}-$ $20 \log _{10} A_{k, s}(x, y)$ and $\theta=\left[x, y, \alpha_{1}, \alpha_{2}, \ldots, \alpha_{k}\right]^{T}$. Subsequently, the gradients of $g_{k, s}(\theta)$ can be computed as:

$$
\begin{aligned}
\frac{\partial g_{k, s}(\theta)}{\partial x} & =-\frac{10 \alpha_{k}}{\ln 10} \frac{u_{k x}}{d_{k}}-\frac{20}{\ln 10} \frac{\partial \ln A_{k, s}(x, y)}{\partial x} \\
\frac{\partial g_{k, s}(\theta)}{\partial y} & =-\frac{10 \alpha_{k}}{\ln 10} \frac{u_{k y}}{d_{k}}-\frac{20}{\ln 10} \frac{\partial \ln A_{k, s}(x, y)}{\partial y} \\
\frac{\partial g_{k, s}(\theta)}{\partial \alpha_{1}} & =\left(\frac{10}{\ln 10} \ln d_{1}\right) \delta_{k 1} \\
\frac{\partial g_{k, s}(\theta)}{\partial \alpha_{2}} & =\left(\frac{10}{\ln 10} \ln d_{2}\right) \delta_{k 2} \\
\vdots & \\
\frac{\partial g_{k, s}(\theta)}{\partial \alpha_{l}} & =\left(\frac{10}{\ln 10} \ln d_{k}\right) \delta_{k l}
\end{aligned}
$$

where $u_{k x}=\frac{x_{k}-x}{d_{k}}, u_{k y}=\frac{y_{k}-y}{d_{k}}, \delta_{k l}=1$ if $k=l$, and $\delta_{k l}=0$, otherwise.

The $(k+2) \times(k+2)$ Fisher information matrix can be represented as follows:

$$
\mathbf{F}_{k+2, k+2}=\left[\begin{array}{cccc}
F_{1,1} & F_{1,2} & \cdots & F_{1, k+2} \\
F_{2,1} & F_{2,2} & \cdots & F_{2, k+2} \\
\vdots & \vdots & \ddots & \vdots \\
F_{k+2,1} & F_{k+2,2} & \cdots & F_{k+2, k+2}
\end{array}\right] .
$$

In the next section, we further define quantitative performance measures for location estimators based on CRB.

\subsection{Accuracy measures}

Let $(\hat{x}, \hat{y})$ be any unbiased location estimator. Then CRB in (22) provides a lower bound on the variance of the unbiased estimator $(\hat{x}, \hat{y})$, that is,

$$
E\left[(\hat{x}-x)^{2}\right] \geq\left[\mathbf{F}^{-1}\right]_{11}, \quad E\left[(\hat{y}-y)^{2}\right] \geq\left[\mathbf{F}^{-1}\right]_{22} .
$$

In location estimation applications, a more meaningful performance measure of location estimators is based on the geometric location estimation error $\epsilon=$ $\sqrt{\left(\hat{x}-x_{k}\right)^{2}+\left(\hat{y}-y_{k}\right)^{2}}$. The mean-squared error (MSE) of any unbiased location estimator is lower bounded as (26) describes:

$$
\epsilon_{\mathrm{rms}}^{2}=E\left[\left(\epsilon^{2}\right] \geq\left[\mathbf{F}^{-1}\right]_{11}+\left[\mathbf{F}^{-1}\right]_{22},\right.
$$

where $\epsilon_{\mathrm{rms}}$ is defined as the root-MSE (RMSE) of location estimators.

Since we assume that the path loss exponent value for each base station is independent of each other, the elements of the Fisher information matrix that include the product of partial derivatives of distinct $\alpha$ values reduce to zero. Let $\tilde{F}_{x}$ be the $(k+1) \times(k+1)$ special matrix obtained by deleting the first row and column of $\mathbf{F}$, i.e.,

$$
\tilde{\mathbf{F}}_{x}=\left[\begin{array}{cccccc}
a & f_{1} & f_{2} & f_{3} & \cdots & f_{n} \\
e_{1} & d_{1} & 0 & 0 & \cdots & 0 \\
e_{2} & 0 & d_{2} & 0 & \cdots & 0 \\
\vdots & \vdots & \ddots & \ddots & \ddots & \vdots \\
e_{n} & 0 & 0 & \cdots & 0 & d_{n}
\end{array}\right]
$$

where its components that might be nonzero are shown with parameters $a, d_{i}, e_{i}$, and $f_{i}, i=1, \ldots, n$. Similarly, let $\tilde{F}_{y}$ be the same type of special matrix obtained by deleting the second row and column of F. Subsequently, (27) can be rewritten as:

$$
\epsilon_{\mathrm{rms}}^{2}=E\left[\epsilon^{2}\right] \geq\left[\mathbf{F}^{-1}\right]_{1,1}+\left[\mathbf{F}^{-1}\right]_{2,2}=\frac{\operatorname{det} \tilde{F}_{x}}{\operatorname{det}(F)}+\frac{\operatorname{det} \tilde{F}_{y}}{\operatorname{det}(F)},
$$


where $\operatorname{det} \tilde{\mathbf{F}}_{x}$ (and similarly $\operatorname{det} \tilde{\mathbf{F}}_{y}$ ) can be computed as [20]:

$$
\operatorname{det} \tilde{\mathbf{F}}_{x}=\prod_{n=1}^{N} d_{n} \cdot\left(a-\sum_{n=1}^{N} \frac{f_{n} e_{n}}{d_{n}}\right)
$$

For the case of three base stations with unequal path loss exponents, the FIM matrices are of size $5 \times 5$ and the unknown vector parameter is given by $\theta=$ $\left[x, y, \alpha_{1}, \alpha_{2}, \alpha_{3}\right]^{T}$. After some algebraic manipulation, $\left[F^{-1}\right]_{11}$ and $\left[F^{-1}\right]_{22}$ can be expressed as:

$$
\begin{aligned}
{\left[\mathbf{F}^{-1}\right]_{11}=} & \left(F_{22} F_{33} F_{44} F_{55}-F_{23}^{2} F_{44} F_{55}\right. \\
& \left.-F_{24}^{2} F_{33} F_{55}-F_{25}^{2} F_{33} F_{44}\right) /|\mathbf{F}| \\
{\left[\mathbf{F}^{-1}\right]_{22}=} & \left(F_{11} F_{33} F_{44} F_{55}-F_{13}^{2} F_{44} F_{55}\right. \\
& \left.-F_{14}^{2} F_{33} F_{55}-F_{15}^{2} F_{33} F_{44}\right) /|\mathbf{F}|,
\end{aligned}
$$

where $|\mathbf{F}|$ is the determinant of the Fisher information matrix and $\epsilon_{\mathrm{rms}}$ is defined as the RMSE of location estimators. The closed form expression of the CRB bound is not explicitly given here due to its complexity; instead, only the numerical solutions are presented. On the other hand, each component of the FIM matrix is given in the Appendix.

\section{Simulation results}

In this section, simulation results for various scenarios are presented and discussed. We assume that all BSs in the network have a three-sector configuration where each sector belongs to a cell with identical coverage area and transmit power. The considered network is composed of three BSs as shown in Figure 2. The cell radius is assumed to be $1 \mathrm{~km}$ for all cells. Sectoral antennas are modeled by the antenna model described in Section 2. BSs are located at Cartesian coordinates [0, 0], [1500, 0], and [0, 1500] in meters. In order to evaluate the effect of the restrictions mentioned in the GSM and UMTS specifications on the performance of the proposed algorithm, two cases for the RSS measurements are considered separately. In the first case, exact measurements are used in the algorithm. In the second case, measurements are truncated if they are below or above the threshold values mentioned in the standards. In the simulations, it is assumed that BS located at coordinates $[0,0]$ is the serving cell and the MS does not change its serving cell (i.e., no handover occurs). At each realization, the MS point MS $=[x, y]$ is generated randomly with a uniform distribution inside the area in the first quadrant of the Cartesian coordinates which is bounded by a circle centered at $[0,0]$ as in Figure 2 .

The proposed algorithm computes the ML estimate of the MS position using the RSS measurements and concurrently calibrates the PLE parameters of the channels occupied by different BSs. Recall that this algorithm is referred to as RSS-MPLE algorithm. In order to demonstrate the improvement on the positioning accuracy provided by the RSS-MPLE algorithm, its performance is compared with those of other algorithms, such as RSS with single PLE algorithm (RSS-SPLE) [46], which finds and calibrates a single PLE for all channels, and RSS with known PLE algorithm (RSS-KPLE) [18] in which PLE values are known as a priori. Furthermore, the Cramer-Rao bound has been evaluated and compared with the RMSE results of the proposed algorithm.

\subsection{Effect of truncated RSS measurements}

RSS measurements below $-110 \mathrm{dBm}$ and above $-48 \mathrm{dBm}$ are truncated in GSM systems. In Figure 3, the effect of such truncation of RSS measurements in the performance of the proposed algorithm is investigated. The case in which all BSs have the same PLE value, i.e., $\alpha_{1}=\alpha_{2}=\alpha_{3}=3$, is considered in this simulation, during which RSS-MPLE and RSS-KPLE algorithms are operated both with truncated and original RSS measurements. The truncated measurements represent all RSS measurements below $-110 \mathrm{dBm}$ and above $-48 \mathrm{dBm}$. In Figure 3 (and the subsequent figures in the paper), the following legend clarification is necessary to better interpret the results:

- 'Truncated RSS are omitted' means that RSS measurement are truncated and measurements below $-110 \mathrm{dBm}$ and above $-48 \mathrm{dBm}$ are not used in the simulations.

- 'Truncated RSS are used' means that RSS measurements being truncated and measurements below $-110 \mathrm{dBm}$ and above $-48 \mathrm{dBm}$ are used in the simulations.

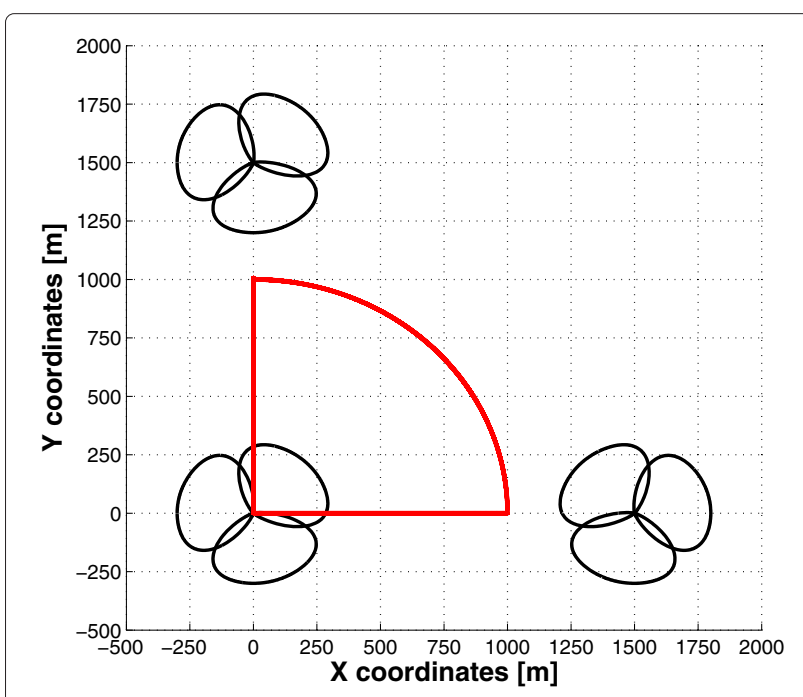

Figure 2 Hypothetical network scheme used throughout the simulations. MS positions are randomly distributed inside the circle on the first quadrant. 


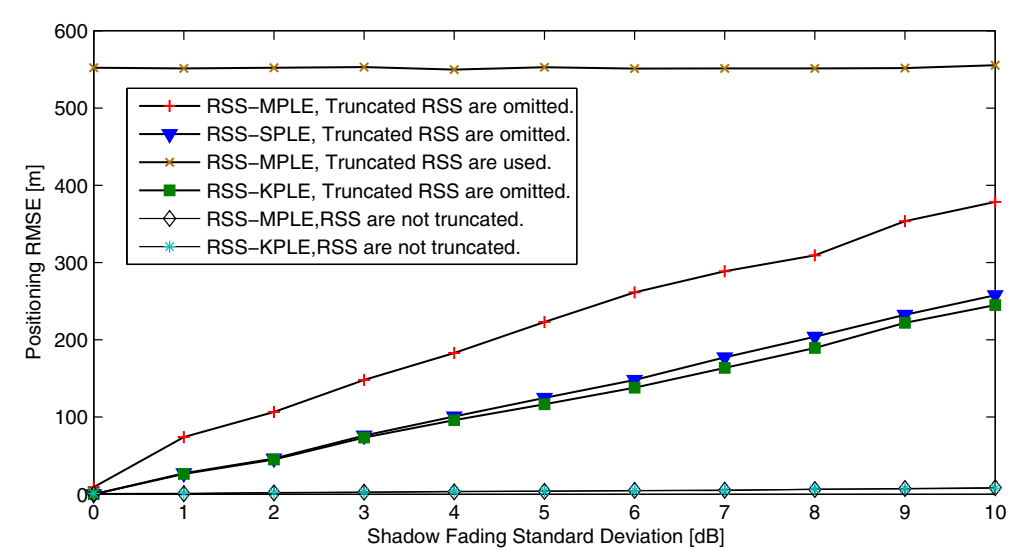

Figure 3 Effect of RSS truncation on position RMSE. When PLE values for all BSs are 3 for $\sigma_{v}$ between 0 to 10 .

- 'RSS are not truncated' means that RSS measurements are not truncated.

From Figure 3, we note that the positioning RMSE obtained with RSS-MPLE algorithm does not exceed $20 \mathrm{~m}$ even for $\sigma_{v}=10$ if RSS measurements are not truncated. On the other hand, truncation of RSS measurements dramatically degrades the performance of both RSS-MPLE and RSS-KPLE algorithms due to the decrease in the number of RSS measurements, i.e., the performance of the proposed algorithm is expected to improve with an increase in the number of available measurements.

Since the truncated measurements represent all RSS measurements below $-110 \mathrm{dBm}$ and above $-48 \mathrm{dBm}$, they introduce a large bias in the position estimate when incorporated in the RSS measurement set. Because of this, positioning accuracy of the RSS-MPLE algorithm severely degrades when the truncated RSS measurements are not omitted. Compared to the RSS-MPLE algorithm, the RSS-KPLE algorithm performs better for all $\sigma_{v}$ values when truncated RSS measurements are used. This is an expected result since RSS-MPLE algorithm estimates PLE values in addition to the coordinates with the same number of RSS measurements.

\subsection{Effect of inaccurate knowledge of PLE values}

In this subsection, the effect of inaccurate PLE values on positioning accuracy is examined and depicted in Figure 4. Throughout the simulation, the PLE values $\alpha_{1}=3.5, \alpha_{2}=$ 2.7, and $\alpha_{3}=2.3$ are used in the RSS-KPLE algorithm, whereas actual PLE values are $\alpha_{1}=3.8, \alpha_{2}=3.0$, and $\alpha_{3}=2.6$. Simulations are carried out with both truncated and exact RSS measurements. As shown in Figure 4, the RSS-MPLE algorithm outperforms the rivals under mild shadow fading since the proposed algorithm is capable of adapting PLE values in real time. On the other hand, the PLE inaccuracy has a drastic effect on positioning accuracy of RSS-KLPLE. As $\sigma_{v}$ increases, the adverse effect of

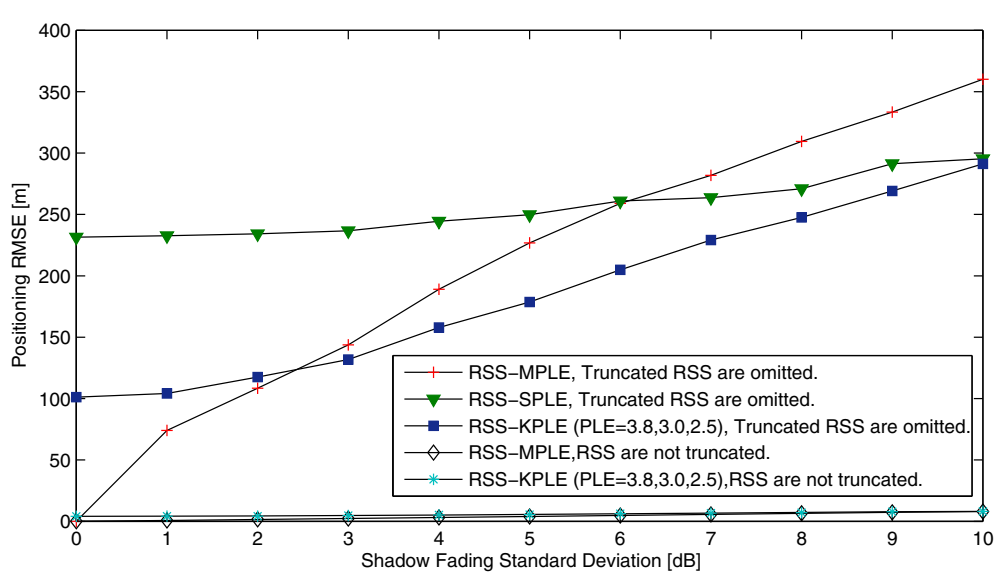

Figure 4 Effect of inaccurate PLEs on position RMSE. $\alpha_{1}=3.5, \alpha_{2}=2.7$, and $\alpha_{3}=2.3$ are used in RSS-KPLE, whereas actual PLES are $\alpha_{1}=3.8, \alpha_{2}=3.0$, and $\alpha_{3}=2.5$. 


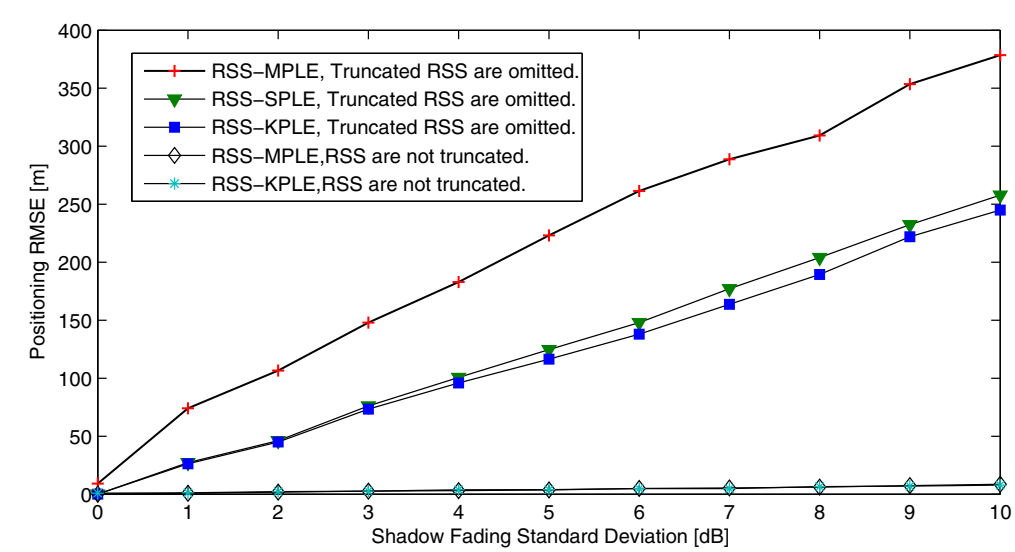

Figure 5 Position RMSE in meters when PLE values for all BSs are 3 for $\sigma_{v}$ between 0 to $10 \mathrm{~dB}$.

the inaccurate PLE values diminishes since shadow fading becomes the main error source.

\subsection{Effect of distinct PLE values}

This subsection focuses on the performance analysis of RSS-MPLE, RSS-SPLE, and RSS-KPLE algorithms under different channel conditions. In the first scenario, it is assumed that all channels have the same PLE value. In the second scenario, PLE values differ for channels occupied by different BSs.

\subsubsection{Equal $\alpha_{1}, \alpha_{2}$, and $\alpha_{3}$}

Let us first consider the rather unrealistic case where all BSs (i.e., all channels) are assumed to have the same PLE value which is equal to three (see Figures 5 and 6). In Figure 5, the RMSE for the positioning algorithms of interest is shown. Since PLE values of all channels are identical, the RSS-SPLE algorithm is expected to outperform the RSS-MPLE algorithm for the same number of

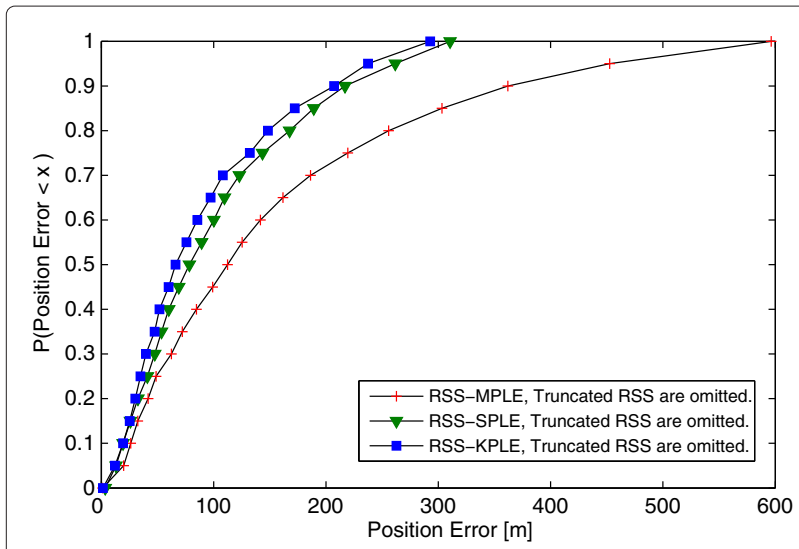

Figure $6 \mathrm{CDF}$ of positioning error for $\sigma_{v}=6 \mathrm{~dB}$ with PLE values of $\alpha_{1}=3, \alpha_{2}=3$, and $\alpha_{3}=3$.
RSS measurements, which is indeed the case as depicted in Figure 5.

To evaluate the RSS-MPLE and RSS-SPLE algorithms with respect to the FCC requirements, the positioning error cumulative distribution function (CDF) is shown in Figure 6 for $\sigma_{v}=6 \mathrm{~dB}$, which is a realistic value in a microcellular environment [31]. The RSS-SPLE and RSS-KPLE algorithms satisfy the FCC requirements, which mandate 67\% CERP within $100 \mathrm{~m}$ and 95\% CERP within $300 \mathrm{~m}$. Although the RSS-MPLE algorithm does not satisfy the FCC requirements, this algorithm offers a solution for environments that possess distinct and variable PLEs.

\subsubsection{Distinct $\alpha_{1}, \alpha_{2}$, and $\alpha_{3}$}

From Figure 5 , it is seen that the RSS-SPLE algorithm has good performance when all PLEs are equal. However, if BSs have different $\alpha$ values, the RSS-MPLE algorithm is expected to outperform the rival algorithms since each PLE is treated separately in the RSS-MPLE algorithm (see Figures 7 and 8). Moreover, as $\sigma_{v}$ increases, the gap between the positioning RMSE of the RSS-MPLE and RSS-SPLE algorithms closes since the error variance of the $\alpha$ estimates obtained with RSS-MPLE algorithm increases. Such a scenario is simulated for BSs that have different PLE values, i.e., for $\alpha_{1}=3.5, \alpha_{2}=2.7$, and $\alpha_{3}=2.3$.

Figure 7 shows that the performance of the RSS-SPLE algorithm deteriorates when PLE values of the BSs are unequal. The scenario considered in this simulation can be experienced when a BS that is in the vicinity of the MS is in NLOS condition and other BSs are in LOS condition with the MS. Compared to the RSS-SPLE algorithm, positioning accuracy of the RSS-MPLE algorithm does not change significantly under these conditions. On the other hand, position estimates obtained with RSS-SPLE algorithm are erroneous due to the bias in the $\alpha$ estimate. Moreover, positioning accuracy of RSS-MPLE and 


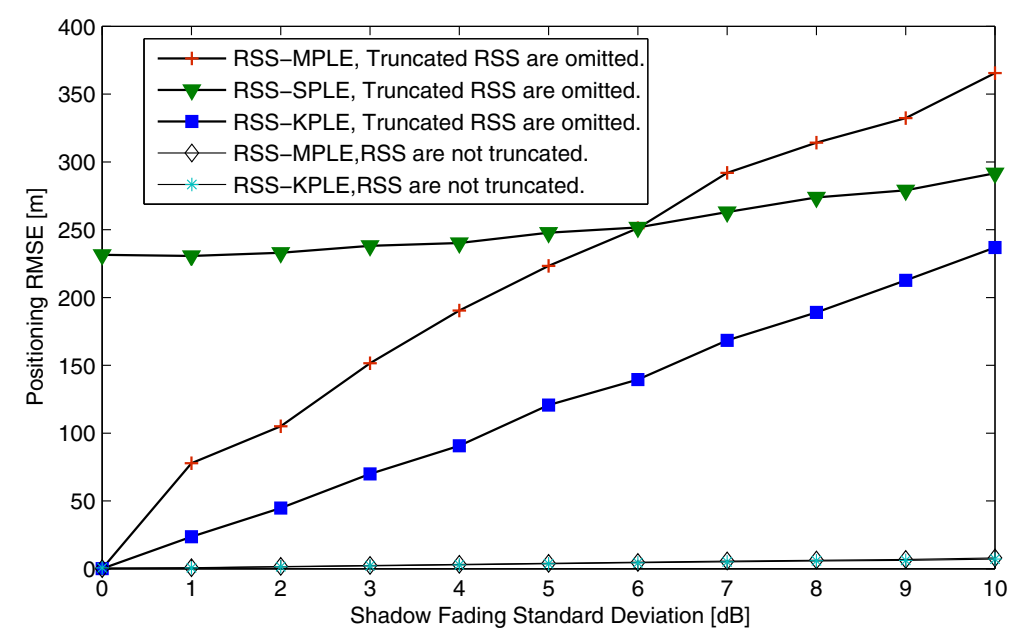

Figure 7 Position RMSE with PLE values. $\alpha_{1}=3.5, \alpha_{2}=2.7$, and $\alpha_{3}=2.3$.

RSS-KPLE algorithms are close even when PLE values vary. Thus, RSS-MPLE algorithm satisfies the requirements for a position estimate with low error variance, independent from unknown propagation parameters, i.e., $\sigma_{v}$ and $\alpha$ values. The positioning error CDF shown in Figure 8 indicates that the accuracy of mobile positioning can substantially be improved by employing the RSSMPLE algorithm.

\subsection{CRB performance evaluation}

In this subsection, we evaluate the CRB bound for the equal PLE case and depict the positioning RMSE for the proposed RSS-MPLE algorithm in Figure 9. From Figure 9, it is noted that the RMSE is relatively high in the middle area, while it shows better results at boundary areas. Actually, this is expected due to the pattern contribution in location estimation since more signal measurements are available for such cases. Figure 10 depicts the CRB results for the same scenario of the proposed algorithm RSS-MPLE. From Figures 9 and 10, it can be concluded that the performance degrades up to $450 \mathrm{~m}$ in RMSE and $400 \mathrm{~m}$ in CRB. Moreover, it is clearly seen that the radiation pattern has a significant effect on location estimation.

\section{Conclusions}

In this paper, a practical positioning method that can be implemented in mobile networks with simple modifications in the existing infrastructure is presented. The proposed method, the so-called RSS-MPLE, is based on RSS measurements and jointly estimates the MS position and the propagation parameters, namely the PLE value of

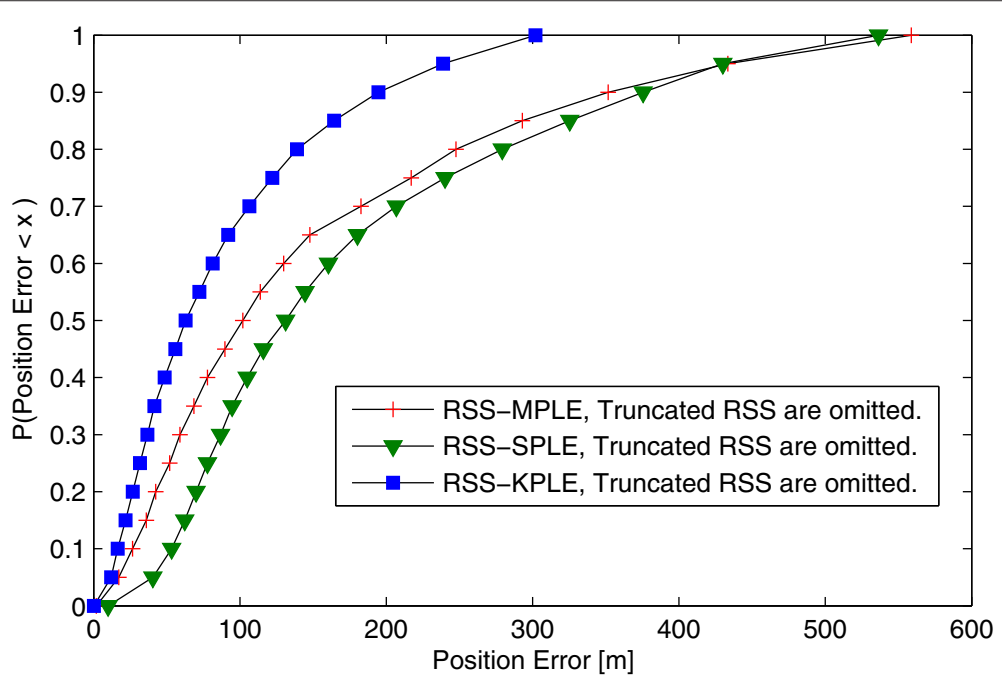

Figure $8 \mathrm{CDF}$ of positioning error for $\sigma_{v}=6 \mathrm{~dB}$ with PLE values of $\alpha_{1}=3.5, \alpha_{2}=2.7$, and $\alpha_{3}=2.3$. 


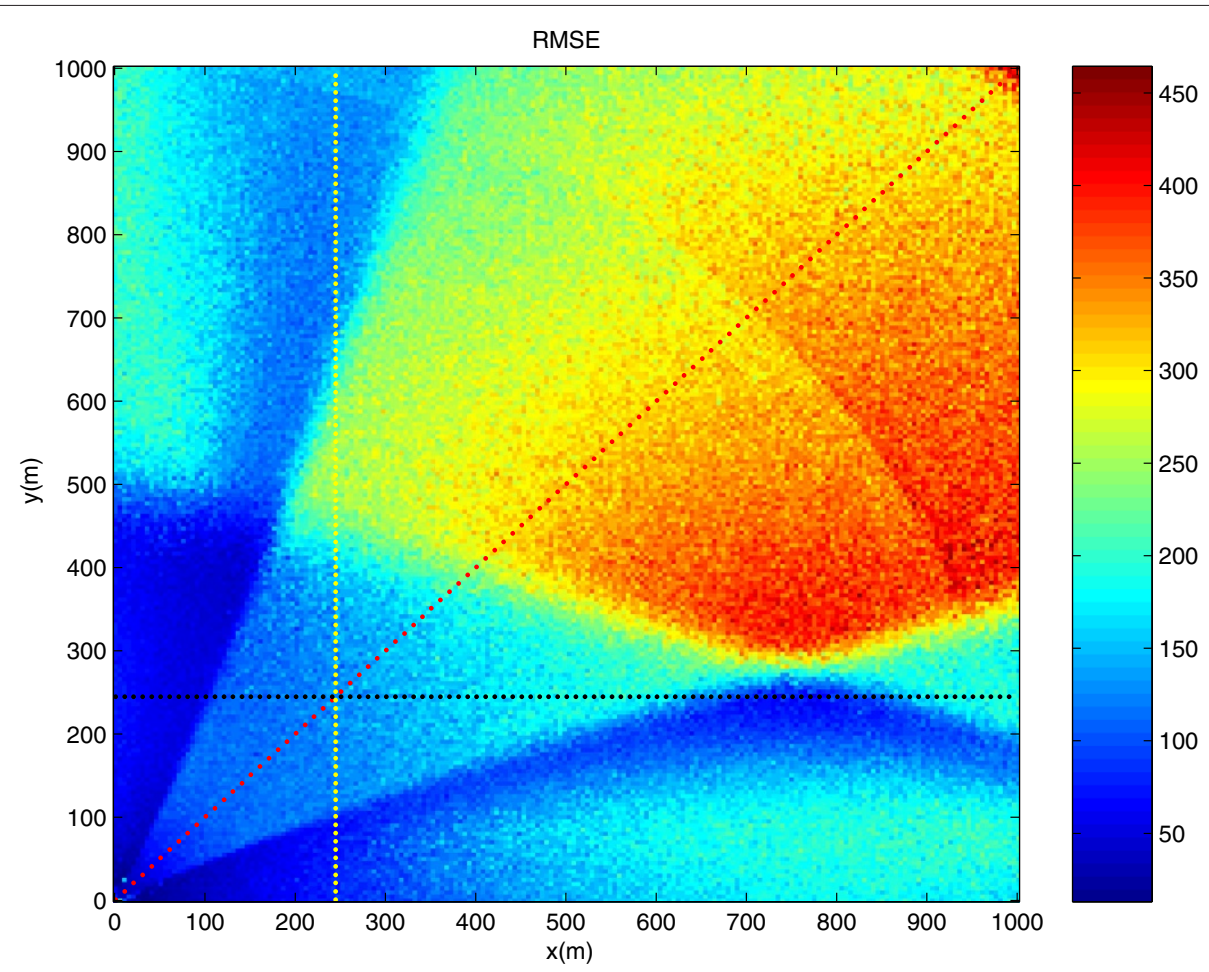

Figure 9 Positioning RMSE in meters while $\alpha=3$ for all BSs.

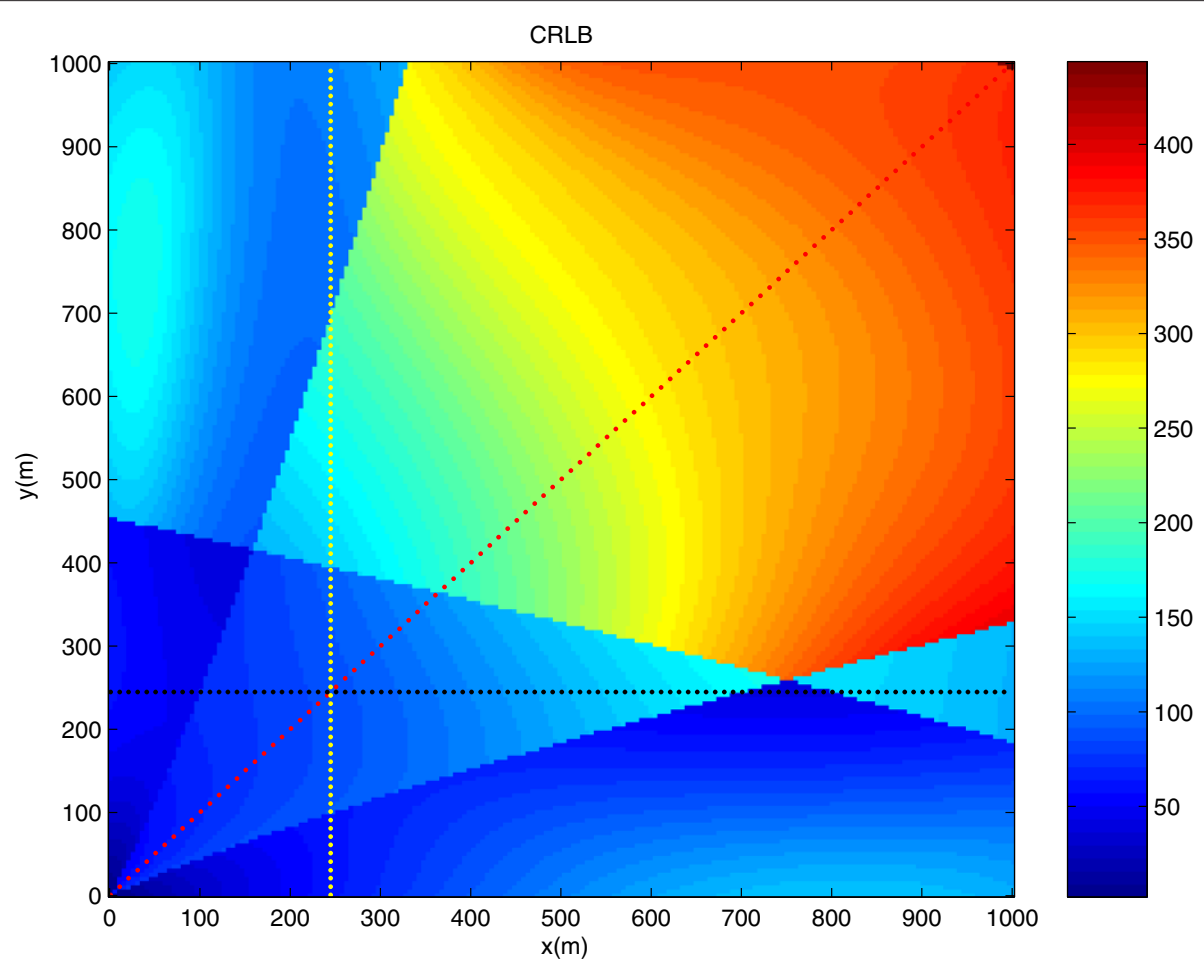

Figure 10 Cramer-Rao bound in meters when $\alpha=3$ for all BSs. 
the measurement channel. The RSS-MPLE method does not need a training period to estimate the PLE value of the channel. The most significant feature of the proposed RSS-MPLE algorithm is its ability to separately calibrate the PLE value of each channel occupied by different BSs. Moreover, the RSS-MPLE algorithm incorporates the antenna radiation pattern information that provides additional improvement in positioning accuracy. Via extensive simulations, the performance of the proposed method has been compared with those of the existing algorithms in terms of positioning RMSE, bias, availability, and CERP under different environmental conditions by changing PLE and SNR values. Simulation results indicate that the RSS-MPLE algorithm is robust against variations in the PLE values under different environment conditions.

\section{Appendix}

\subsection{Cramer-Rao bound for the three BS case}

The components of the Fisher information matrix are given as below:

$$
\begin{aligned}
& F_{11}=\frac{1}{\sigma_{v}^{2}} \sum_{k=1}^{n} \sum_{s=1}^{3}\left(\frac{\partial g_{k, s}(\theta)}{\partial x}\right)^{2} \\
& =\frac{1}{\sigma_{v}^{2}} \sum_{k=1}^{n} \sum_{s=1}^{3}\left(\frac{-10 \alpha_{k}}{\ln 10} \frac{u_{k x}}{d_{k}}-\frac{20}{\ln 10} \frac{\partial \ln A_{k, s}(x, y)}{\partial x}\right)^{2} \\
& F_{12}=\frac{1}{\sigma_{v}^{2}} \sum_{k=1}^{n} \sum_{s=1}^{3}\left(\frac{\partial g_{k, s}(\theta)}{\partial x}\right)\left(\frac{\partial g_{k, s}(\theta)}{\partial y}\right) \\
& =\frac{1}{\sigma_{v}^{2}} \sum_{k=1}^{n} \sum_{s=1}^{3}\left(\frac{-10 \alpha_{k}}{\ln 10} \frac{u_{k x}}{d_{k}}-\frac{20}{\ln 10} \frac{\partial \ln A_{k, s}(x, y)}{\partial x}\right) \\
& \left(\frac{-10 \alpha_{k}}{\ln 10} \frac{u_{k y}}{d_{k}}-\frac{20}{\ln 10} \frac{\partial \ln A_{k, s}(x, y)}{\partial y}\right) \\
& F_{13}=\frac{1}{\sigma_{v}^{2}} \sum_{k=1}^{n} \sum_{s=1}^{3}\left(\frac{\partial g_{k, s}(\theta)}{\partial x}\right)\left(\frac{\partial g_{k, s}(\theta)}{\partial \alpha_{1}}\right) \\
& =\frac{1}{\sigma_{v}^{2}} \sum_{s=1}^{3}\left(\frac{-10 \alpha_{1}}{\ln 10} \frac{u_{1 x}}{d_{1}}-\frac{20}{\ln 10} \frac{\partial \ln A_{1, s}(x, y)}{\partial x}\right)\left(\frac{10}{\ln 10} \ln d_{1}\right) \text {, } \\
& F_{14}=\frac{1}{\sigma_{v}^{2}} \sum_{k=1}^{n} \sum_{s=1}^{3}\left(\frac{\partial g_{k, s}(\theta)}{\partial x}\right)\left(\frac{\partial g_{k, s}(\theta)}{\partial \alpha_{2}}\right) \\
& =\frac{1}{\sigma_{v}^{2}} \sum_{s=1}^{3}\left(\frac{-10 \alpha_{k}}{\ln 10} \frac{u_{2 x}}{d_{2}}-\frac{20}{\ln 10} \frac{\partial \ln A_{2, s}(x, y)}{\partial x}\right)\left(\frac{10}{\ln 10} \ln d_{2}\right) \text {, } \\
& F_{15}=\frac{1}{\sigma_{v}^{2}} \sum_{k=1}^{n} \sum_{s=1}^{3}\left(\frac{\partial g_{k, s}(\theta)}{\partial x}\right)\left(\frac{\partial g_{k, s}(\theta)}{\partial \alpha_{3}}\right) \\
& =\frac{1}{\sigma_{v}^{2}} \sum_{s=1}^{3}\left(\frac{-10 \alpha_{3}}{\ln 10} \frac{u_{3 x}}{d_{3}}-\frac{20}{\ln 10} \frac{\partial \ln A_{3, s}(x, y)}{\partial x}\right)\left(\frac{10}{\ln 10} \ln d_{3}\right) \text {, }
\end{aligned}
$$

$$
\begin{aligned}
& F_{21}=F_{12} \\
& F_{22}=\frac{1}{\sigma_{v}^{2}} \sum_{k=1}^{n} \sum_{s=1}^{3}\left(\frac{\partial g_{k, s}(\theta)}{\partial y}\right)^{2} \\
& =\frac{1}{\sigma_{v}^{2}} \sum_{k=1}^{n} \sum_{s=1}^{3}\left(\frac{-10 \alpha_{k}}{\ln 10} \frac{u_{k y}}{d_{k}}-\frac{20}{\ln 10} \frac{\partial \ln A_{k, s}(x, y)}{\partial y}\right)^{2} \\
& F_{23}=\frac{1}{\sigma_{v}^{2}} \sum_{k=1}^{n} \sum_{s=1}^{3}\left(\frac{\partial g_{k, s}(\theta)}{\partial y}\right)\left(\frac{\partial g_{k, s}(\theta)}{\partial \alpha_{k}}\right) \\
& =\frac{1}{\sigma_{v}^{2}} \sum_{s=1}^{3}\left(\frac{-10 \alpha_{1}}{\ln 10} \frac{u_{1 y}}{d_{1}}-\frac{20}{\ln 10} \frac{\partial \ln A_{1, s}(x, y)}{\partial y}\right)\left(\frac{10}{\ln 10} \ln d_{1}\right), \\
& F_{24}=\frac{1}{\sigma_{v}^{2}} \sum_{k=1}^{s} \sum_{s=1}^{3}\left(\frac{\partial g_{k, s}(\theta)}{\partial y}\right)\left(\frac{\partial g_{k, s}(\theta)}{\partial \alpha_{2}}\right) \\
& =\frac{1}{\sigma_{v}^{2}} \sum_{s=1}^{3}\left(\frac{-10 \alpha_{2}}{\ln 10} \frac{u_{2 y}}{d_{2}}-\frac{20}{\ln 10} \frac{\partial \ln A_{2, s}(x, y)}{\partial y}\right)\left(\frac{10}{\ln 10} \ln d_{2}\right) \text {, } \\
& F_{25}=\frac{1}{\sigma_{v}^{2}} \sum_{k=1}^{n} \sum_{s=1}^{3}\left(\frac{\partial g_{k, s}(\theta)}{\partial y}\right)\left(\frac{\partial g_{k, s}(\theta)}{\partial \alpha_{3}}\right) \\
& =\frac{1}{\sigma_{v}^{2}} \sum_{s=1}^{3}\left(\frac{-10 \alpha_{3}}{\ln 10} \frac{u_{3 y}}{d_{3}}-\frac{20}{\ln 10} \frac{\partial \ln A_{3, s}(x, y)}{\partial y}\right)\left(\frac{10}{\ln 10} \ln d_{3}\right) \text {, } \\
& F_{31}=F_{13} \\
& F_{32}=F_{23} \\
& F_{33}=\frac{1}{\sigma_{v}^{2}} \sum_{k=1}^{n} \sum_{s=1}^{3}\left(\frac{\partial g_{k, s}(\theta)}{\partial \alpha_{1}}\right)\left(\frac{\partial g_{k, s}(\theta)}{\partial \alpha_{1}}\right) \\
& =\frac{1}{\sigma_{v}^{2}} \sum_{s=1}^{3}\left(\frac{10}{\ln 10} \ln d_{1}\right)^{2} \\
& F_{34}=0, F_{35}=0 \\
& F_{41}=F_{14}, F_{42}=F_{24} \\
& F_{44}=\frac{1}{\sigma_{v}^{2}} \sum_{k=1}^{n} \sum_{s=1}^{3}\left(\frac{\partial g_{k, s}(\theta)}{\partial y}\right)\left(\frac{\partial g_{k, s}(\theta)}{\partial \alpha_{2}}\right) \\
& =\frac{1}{\sigma_{v}^{2}} \sum_{s=1}^{3}\left(\frac{10}{\ln 10} \ln d_{2}\right)^{2} \\
& F_{43}=0, F_{45}=0 \\
& F_{51}=F_{15}, F_{52}=F_{25} \\
& F_{53}=F_{35}=0, F_{54}=F_{45}=0 \\
& F_{55}=\frac{1}{\sigma_{v}^{2}} \sum_{k=1}^{n} \sum_{s=1}^{3}\left(\frac{\partial g_{k, s}(\theta)}{\partial \alpha_{3}}\right)\left(\frac{\partial g_{k, s}(\theta)}{\partial \alpha_{3}}\right) \\
& =\frac{1}{\sigma_{v}^{2}} \sum_{s=1}^{3}\left(\frac{10}{\ln 10} \ln d_{3}\right)^{2}
\end{aligned}
$$

Competing interests

The authors declare that they have no competing interests. 


\section{Acknowledgements}

The authors would like to thank the referees for their constructive comments which improved the exposition of the paper.

The authors dedicate this article in memory of one of its co-authors, Frederic Kerem Harmanci, who passed away between the completion of his article and its publication.

\section{Received: 13 October 2012 Accepted: 16 June 2013}

Published: 26 June 2013

\section{References}

1. JJ Caffery, Wireless Location in CDMA Cellular Radio Systems. (Springer, Heidelberg, 2000)

2. IEEE P802.15.4a/D4, Part 15.4: Wireless Medium Access Control(MAC) and Physical Layer (PHY) Specifications for Low-Rate wireless personal area networks (LR-WPANs): Amendment of IEEE Std 802.15.4, July 2006. (IEEE, Piscataway, 2006)

3. FC Commission, Revision of the Commission's Rules to Ensure Compatibility with Enhanced 911 Emergency Calling Systems, FCC 94-102. (FC Commission, Washington DC, 1996)

4. J Borkowski, J Niemela, J Lempiäinen, Cellular location techniques supporting A-GPS positioning. IEEE Vehicular Technol. Conf. 1, 429-433 (2005)

5. F Gustafsson, F Gunnarsson, Mobile positioning using wireless networks: Possibilities and fundamental limitations based on available wireless network measurements. IEEE Signal Process. Mag. 22(4), 41-53 (2005)

6. K Cheung, H So, W-K Ma, Y Chan, Least squares algorithms for time-of-arrival-based mobile location. IEEE Trans. Signal Process. 52(4), 1121-1130 (2004)

7. BJ Dil, PJM Havinga, in Australasian Telecommunication Networks and Applications Conference, ATNAC 2010 (Auckland, 31 October 3 November 2010)

8. B Lakmali, D Dias, Database correlation for GSM location in outdoor \& indoor environments. The 4th International Conference on Information and Automation for Sustainability, Colombo, 12-14 December 2008. (IEEE, Piscataway, 2008), pp. 42-47

9. S Ahonen, $\mathrm{H}$ Laitinen, Database correlation method for UMTD location. IEEE Vehicular Technol. Conf. 4, 2696-2700 (2003)

10. M Spirito, A Mattioli, Preliminary experimental results of a GSM mobile phones positioning system based on timing advance. IEEE Vehicular Technol. Conf. 4, 2072-2076 (1999)

11. M Spirito, On the accuracy of cellular mobile station location estimation. IEEE Trans. Vehicular Technol. 50(3), 674-685 (2001)

12. M Khalaf-Allah, Nonparametric Bayesian filtering for location estimation, position tracking and global localization of mobile terminals in outdoor wireless environments. EURASIP J Adv. Signal Process. 2008, 1-14 (2008)

13. J Borkowski, J Lempiäinen, Pilot correlation positioning method for urban UMTS networks. Proceedings of the 11th European Wireless Conference, Nicosia, 10-13 April 2005. vol. 2. (IEEE, Piscataway, 2005), pp. 465-469

14. W Pan, J Wu, Z Jiang, Y Wang, X You, Mobile position tracking by TDOA-Doppler hybrid estimation in mobile cellular system, IEEE International Conference on Communications, Glasgow, 24-28 June 2007. (IEEE, Piscataway, 2007), pp. 4670-4673

15. O Türkyilmaz, F Alagöz, G Gür, T Tugcu, Environment-aware location estimation in cellular networks. EURASIP J. Adv. Signal Process. 2008, 276456 (2008)

16. A Sayed, A Tarighat, N Khajehnouri, Network-based wireless location: challenges faced in developing techniques for accurate wireless location information. IEEE Signal Process. Mag. 22(4), 24-40 (2005)

17. J Borkowski, J Lempiäinen, Practical network-based techniques for mobile positioning in UMTS. EURASIP J. Appl. Signal Process. 2006, 012930 (2006)

18. K Budka, D Calin, B Chen, D Jeske, A Bayesian method to improve mobile geolocation accuracy. IEEE Vehicular Technol. Conf. 2, 1021-1025 (2002)

19. KMK Chu, KRPH Leung, Ng JK-Y, CH Li, Locating mobile stations with statistical directional propagation model in AINA '04. Proceedings of the 18th International Conference on Advanced Information Networking and Applications, Washington, 29-31 March 2004. (IEEE, Piscataway, 2004), pp. 230-235
20. Y Qi, H Kobayashi, Cramer-Rao Lower bound for geolocation in non-line-of-sight environment. IEEE Int. Conf. Acoustics, Speech, Signal Process. (ICASSP). 3, 2473-2476 (2002)

21. R Yamamoto, H Matsutani, H Matsuki, T Oono, H Ohtsuka, Position location technologies using signal strength in cellular systems. IEEE Vehicular Technol. Conf. 4, 2570-2574 (2001)

22. M Aso, T Saikawa, T Hattori, Maximum likelihood location estimation using signal strength and the mobile station velocity in cellular systems. Vehicular Technol. Conf. 2, 742-746 (2003)

23. J Zhou, J-Y Ng, A data fusion approach to mobile location estimation based on ellipse propagation model within a cellular radio network, The 21st International Conference on Advanced Information Networking and Applications Niagara Falls, 21 - 23 May 2007. (IEEE, Piscataway, 2007), pp. 459-466

24. M Akar, U Mitra, Variations on optimal and suboptimal handoff control for wireless communication systems. IEEE J. Selected Areas Commun. 19(6), 1173-1185 (2001)

25. L Mihaylova, D Angelova, S Honary, D Bull, N Canagarajah, B Ristic, Mobility tracking in cellular networks using particle filtering. IEEE Trans. Wireless Commun. 6(10), 3589-3599 (2007)

26. K Achutegui, J Rodas, CJ Escudero, J Miguez, A model-switching sequential Monte Carlo algorithm for indoor tracking with experimental RSS data. The International Conference on Indoor Positioning and Indoor Navigation (IPIN), Zurich, 15-17 September 2010. (IEEE, Piscataway, 2010), pp. 1-8

27. J Turkka, M Renfors, Path loss measurements for a non-line-of-sight mobile-to-mobile environment The 8th International Conference on ITS Telecommunications, Phuket, 24-24 October 2008. (IEEE, Piscataway, 2008), pp. 274-278

28. S Srinivasa, M Haenggi, Path loss exponent estimation in large wireless networks. (CERN Document Server, Meyrin, 2008)

29. J Shirahama, T Ohtsuki, RSS-based localization in environments with different path loss exponent for each link. IEEE Vehicular Technology Conference, Singapore, 11-14 May 2008. (IEEE, Piscataway, 2008), pp. 1509-1513

30. I Popescu, D Nikitopoulos, P Constantinou, I Nafornita, ANN prediction models for outdoor environment. IEEE 17th International Symposium on Personal, Indoor and Mobile Radio Communications, Helsinki, 11-14 September 2006. (IEEE, Piscataway, 2006), pp. 1-5

31. T Rappaport, Wireless Communications. (Prentice Hall PTR, New Jersey, 2002)

32. V Erceg, $\sqcup$ Greenstein, SY Tjandra, SR Parkoff, A Gupta, B Kulic, AA Julius, An empirically based path loss model for wireless channels in suburban environments. IEEE J. Selected Areas Commun. 17(7), 1999-1205 (1999)

33. K Martinez, JK Hart, R Ong, Environmental sensor networks. Computer 37, 50-56 (2004)

34. LJ Greenstein, V Erceg, Gain reductions due to scatter on wireless paths with directional antennas. IEEE Commun. Lett. 3(6), 169-171 (1999)

35. PF Wilson, PB Papazian, MG Cotton, Y Lo, Advanced antenna test bed characterization for wideband wireless communications, NTIA Technical report. (US Department of Commerce, Washington DC, 1999)

36. TA Rahman, GB Ser, Propagation measurement in IMT-2000 band in Malaysia environment. TENCON 2000. 1,77-81 (2000)

37. S-W Wang, I Wang, Effects of soft handoff, frequency reuse and non-ideal antenna sectorization on CDMA system capacity. 43rd IEEE Vehicular Technology Conference, Secaucus, 18-20 May 1993. (IEEE, Piscataway, 1993), pp. 850-854

38. A Vavoulas, N Vaiopoulos, DA Varoutas, A Chipouras, G Stefano, Performance improvement of fixed wireless access networks by conjunction of dual polarization and time domain radio resource allocation technique. Int. J Commun. Syst. 24, 483-491 (2010)

39. J Park, JW Jang, S-G Park, W Sung, Capacity of sectorized distributed networks employing adaptive collaboration from remote antennas. IEICE Trans. E93-B, 3534-3537 (2010)

40. D Runyon, Optimum directivity coverage of fan-beam antennas. IEEE Antennas Propagation Mag. 44(2), 66-70 (2002)

41. SM Kay, Fundamentals of Statistical Signal Processing: Estimation Theory. (Prentice Hall PTR, New Jersey, 1993)

42. N Yamashita, M Fukushima, On the rate of convergence of the Levenberg-Marquardt methods. Comput Suppl. 15, 237-249 (2001) 
43. J-Y Fan, Y-X Yuan, On the quadratic convergence of the Levenberg-Marquardt method without nonsingularity assumption. Computing. 74, 23-39 (2005)

44. K Madsen, HB Nielsen, O Tingleff, Methods for Non-linear Least Squares Problems. (Informatics and Mathematical Modelling Technical University of Denmark, Lyngby, 2004)

45. K Pahlavan, A Levesque, Wireless Information Networks. (John Wiley and Sons, Inc., New York, 1995)

46. X Li, RSS-based location estimation with unknown pathloss model. IEEE Trans. Wireless Commun. 5(12), 3626-3633 (2006)

doi:10.1186/1687-1499-2013-178

Cite this article as: Zeytinci et al:: Location estimation using RSS measurements with unknown path loss exponents. EURASIP Journal on Wireless Communications and Networking 2013 2013:178.

\section{Submit your manuscript to a SpringerOpen ${ }^{\mathcal{O}}$ journal and benefit from:}

- Convenient online submission

- Rigorous peer review

- Immediate publication on acceptance

- Open access: articles freely available online

- High visibility within the field

- Retaining the copyright to your article

Submit your next manuscript at $\gg$ springeropen.com 